\section{CHIRPED FIBER BRAGG GRATING CAVITY RING-DOWN FOR STRAIN SENSING USING AN OTDR}

\author{
Susana Silva, Daniel J. Passos, Manuel B. Marques, and \\ Orlando Frazão \\ INESC Porto and Department of Physics and Astronomy, Faculty of \\ Science at University of Porto Rua do Campo Alegre, 687, 4169-007 \\ Porto, Portugal; Corresponding author: sfsilva@inescporto.pt
}

\section{Received 31 October 2014}

ABSTRACT: This work presents a fiber cavity ring down (CRD) configuration for the measurement of strain. An optical time-domain reflectometer (OTDR) was used to send impulses down into the fiber loop cavity, inside of which a chirped fiber Bragg grating was placed to act as a strain sensing element. This technique could provide strain results with both conventional CRD-based configuration and the OTDR. The $C R D$ configuration provided a linear response to strain applied in the range 0-2000 $\mu \varepsilon$, and a sensitivity of $1.34 n s / \mu \varepsilon$ was obtained. For the same operation range, the OTDR interrogation allowed obtaining a periodic behavior due to fiber Bragg grating scanning of the wavelength peaks of the multimode laser source while increasing strain. It is shown that the OTDR interrogation method provide a ring-down time response of about $8 \mu$ s wich is a great improvement when compared to the conventional CRD configuration $(23 \mu \mathrm{s})$. (c) 2015 Wiley Periodicals, Inc. Microwave Opt Technol Lett 57:1442-1444, 2015; View this article online at wileyonlinelibrary.com. DOI 10.1002/mop.29114

Key words: cavity ring down; optical time domain reflectometer; optical fiber sensors; strain

\section{INTRODUCTION}

Cavity ring down (CRD) spectroscopy is a highly sensitive spectroscopic technique that measures changes in the decay rate of an optical resonator and it has been in the past few decades a strong subject of research [1]. Although typically implemented in a mirror-based cavity in bulk optics, the CRD is commonly applied to real-time chemical analysis but it is also an effective method for determining the loss in an optical fiber-based loop resonator $[2,3]$.

The appearance of the fiber-optic based CRD, which uses a fiber loop as resonant cavity, emerged as a potential alternative to the usual CRD for spectroscopy; and quickly suggested a range of new applications, including distributed monitoring over a large physical area or even remote sensing. In fact, in the last few years, this technique has been implemented in the measurement of different physical parameters, namely, strain [4,5], pressure [6], temperature [7], refractive index [8-10], and biochemical sensing [11-15].

In this work, a fiber CRD configuration using an optical time domain reflectometer (OTDR) optical modulated source is presented for the measurement of strain. The sensing head, which is placed inside the fiber loop cavity, is based on a chirped fiber Bragg grating (FBG) and acts as a strain sensing element. The strain results were attained with both conventional CRD-based configuration and the OTDR. For the first case, the FBG sensor acts as a band-rejection filter; while for the second case, the FBG is interrogated in reflection.

\section{EXPERIMENTAL RESULTS}

The experimental setup of the proposed CRD chirped-FBG system is presented in Figure 1. The cavity is composed of two standard $(2 \times 1)$ 1:99 optical fiber couplers, a fiber loop with $\sim 1560 \mathrm{~m}$ (SMF 28) and a chirped-FBG. A commercial OTDR
(Yokogawa AQ7270) is used to send impulses (1 $\mu$ s at $1550 \mathrm{~nm}$ ) down into the fiber cavity, instead of the usual laser and modulator setup [16]. The train of pulses is coupled via $1 \%$ arm of the input optical coupler, rings around inside the fiber loop and is coupled out via $1 \%$ arm of the output coupler; the amplitude of the output pulses decay temporally due to the total existing losses in the fiber loop (fiber loss, fiber couplers insertion losses, chirped-FBG transmission attenuation), passes through a photodetector (gain of $40 \mathrm{~dB}$ ) and is monitored in an oscilloscope. The chirped-FBG placed inside the fiber loop cavity is centered at $1573 \mathrm{~nm}$ and it has about $4 \mathrm{~nm}$ width. The proposed technique allows dual interrogation of the chirpedFBG incorporated in a conventional CRD topology. The output signal is read (1) through the oscilloscope or (2) by the OTDR. In the first case, the output signal of the OTDR is used only as optical modulated source [17].

To perform strain measurements, the chirped FBG was fixed at two points that were $200 \mathrm{~mm}$ apart, and submitted to specific strain values by means of a translation stage (via sequential 20 $\mu \mathrm{m}$ displacements). When strain was applied to the chirpedFBG, two distinct output responses could be observed either in the oscilloscope or in the OTDR-see subsections 2.1 and 2.2, respectively.

In overall, the output signal response is strongly dependent of the wavelength position of the FBG with respect to the OTDR laser source. Usually, the optical sources used in the OTDR are multimode lasers centered at $1550 \mathrm{~nm}$. In this case, the FBG in interrogated in transmission (recall Fig. 1). If the chirped-FBG is placed at lower wavelengths, the output signal is expected to decrease with increasing strain. Conversely, if the FBG is placed at wavelengths higher than $1550 \mathrm{~nm}$ (in this case, the FBG is centered at $1570 \mathrm{~nm}$ ) the output signal is expected to increase with applied strain. The following subsections present the results attained by interrogating the chirpedFBG with both conventional CRD configuration and OTDR.

\subsection{Conventional CRD Interrogation}

The decay curve from one cavity ring-down decay is illustrated in Figure 2. The obtained output signal corresponds to a typical loop ring-down waveform of the conventional CRD.

For this configuration, the time of a single round trip is about $7.7 \mu \mathrm{s}$ and is strongly dependent of the cavity length. An exponential fit was performed and a ring-down time of $22.8 \mu \mathrm{s}$ was attained. This value depends strongly on the losses originated by the length of the loop, splices, and insertion losses of the fiber couplers [3].

Figure 3 shows the CRD response with applied strain to the chirped-FBG. In this case, an increase of the ring-down time is observed. A linear response was obtained due to the strain feature of the chirped-FBG (linear response $d \lambda / d \varepsilon$ ) and the sensor presents a sensitivity of $1.34 \mathrm{~ns} / \mu \varepsilon$. The photodetector reads all the light that comes from the OTDR except the light backreflected by the FBG. Recall that the spectral response of the FBG is superimposed with the spectral response of the optical source (OTDR). Therefore, when subjected to strain, the transmission spectrum of the FBG moves outside the central wavelength of the multimode laser of the OTDR (i.e., at $1550 \mathrm{~nm}$ ), providing an amplitude increase of the output power detected by the photodetector. This effect appears when the FBG acts as a band-rejection filter.

\subsection{OTDR Interrogation}

Figure 4 presents the OTDR pulse intensity decay that is backreflected by the chirped-FBG inside the fiber loop. One can 


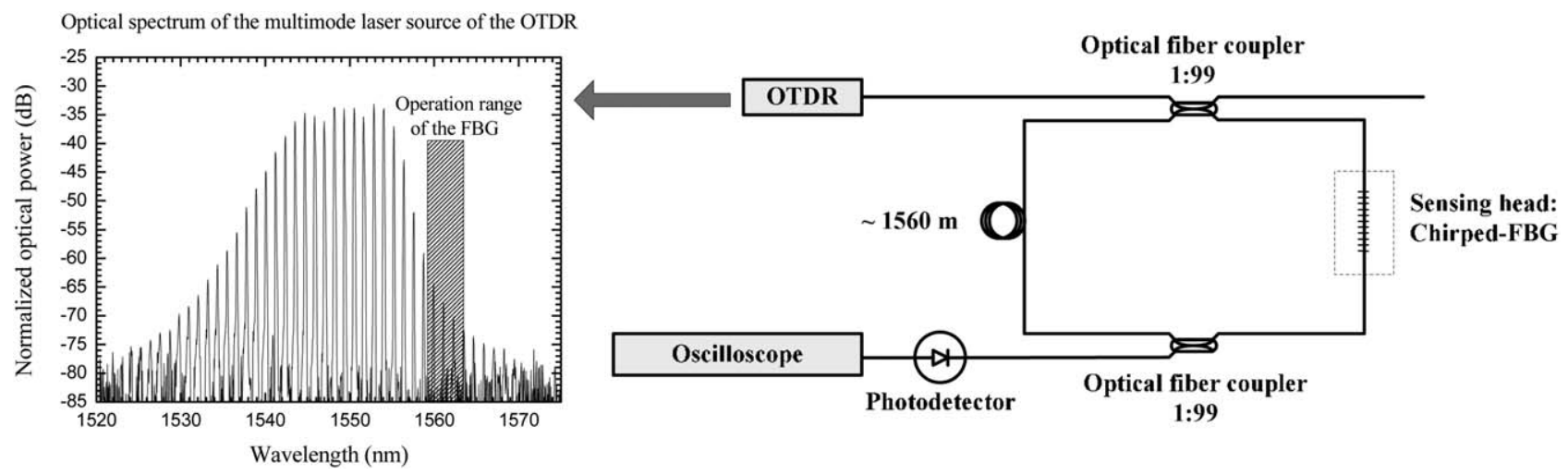

Figure 1 Experimental setup of the proposed CRD chirped-FBG (left side) and optical spectrum of the multimode laser source of the OTDR used in the experiment. The signal is introduced inside the fiber cavity by means of an OTDR and monitored via a photodetector and an oscilloscope

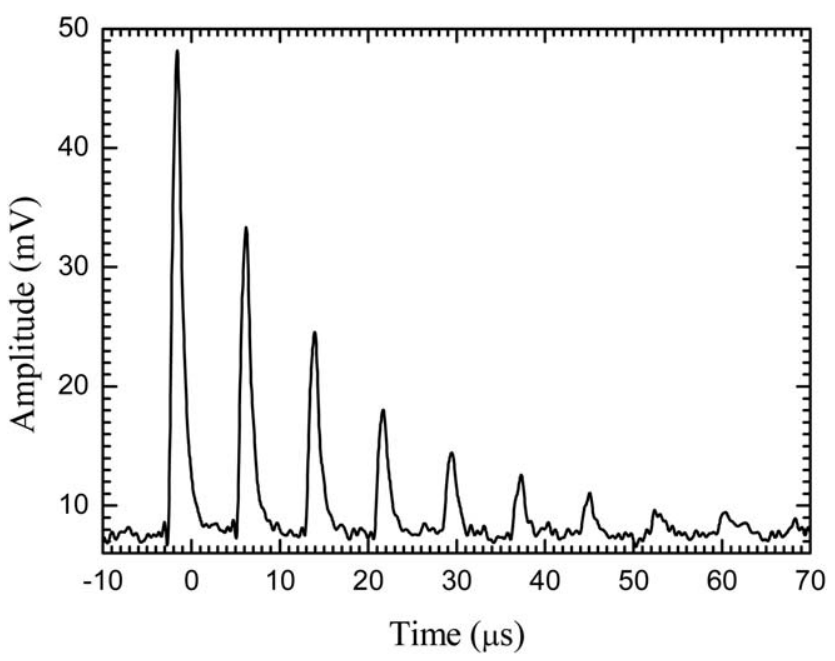

Figure 2 Cavity ring-down decay with a chirped-FBG inside the fiber loop

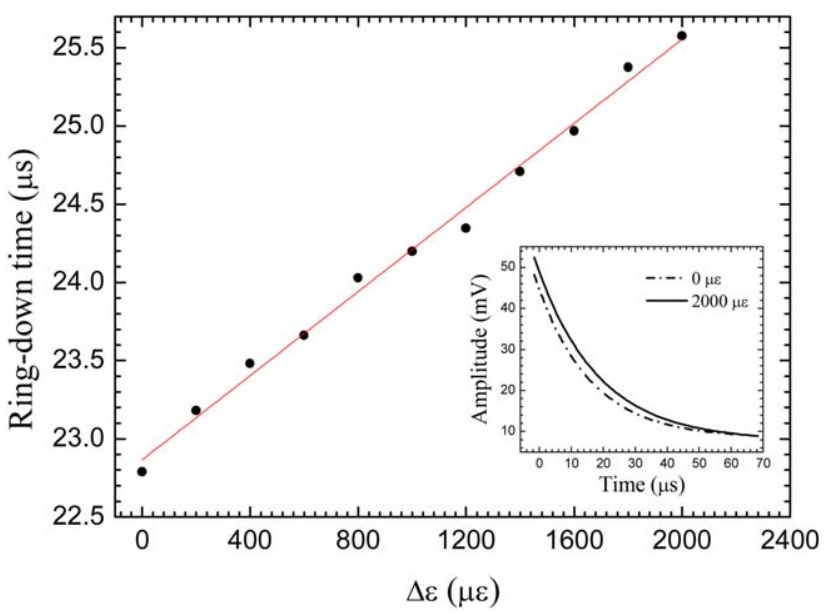

Figure 3 Cavity ring-down time versus applied strain to the chirpedFBG when interrogated by the photodetector. Inset, peak amplitude decay with increasing strain. [Color figure can be viewed in the online issue, which is available at wileyonlinelibrary.com]

observe at the beginning signal saturation that may be avoided by placing a few meters of fiber (SMF 28) between the OTDR and the ring cavity. Afterward, the amplitude of the signal

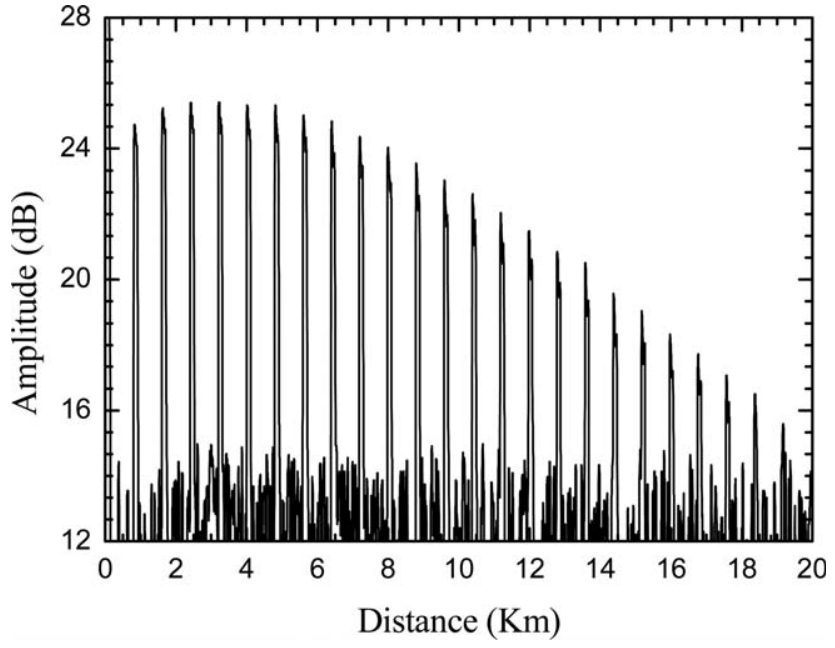

Figure 4 OTDR waveform of the pulse intensity decay of the chirpedFBG inside the fiber loop

decays due to the several round trips of the pulse inside the cavity, as expected.

The operation mode of the cavity ring-down interrogated by the OTDR is based on the second reading of the back-reflected light from the FBG. In this case, the light traveled about $1600 \mathrm{~m}$ which corresponds to a single round trip of $8 \mu \mathrm{s}$, similar to the one obtained with the CRD configuration. This also corresponds to the distance between two consecutive peaks $(800 \mathrm{~m}$ from observing Fig. 4) — due to signal processing of the OTDR, the back-reflected and measured light is divided by 2 ; therefore, the effective distance between two consecutive peaks is about $1600 \mathrm{~m}$.

The OTDR may also be a viable interrogation method as depicted in Figure 5.When strain is applied, the FBG moves to longer wavelengths, scanning the several wavelength peaks of the multimode laser source (recall operation range of the FBG in Fig. 1) and thus resulting in a periodic behavior as shown in Figure 5. In this case, the ring-down time is almost 10-fold the one obtained with the CRD configuration $(22.8 \mu \mathrm{s})$ and ranges from 180 to $206 \mu$ s due to the FBG position regarding the multimode laser source.

\section{CONCLUSION}

A fiber-based CRD configuration using an OTDR as optical modulated source was presented in this work for the measurement of strain. A chirped-FBG was placed inside the fiber loop 


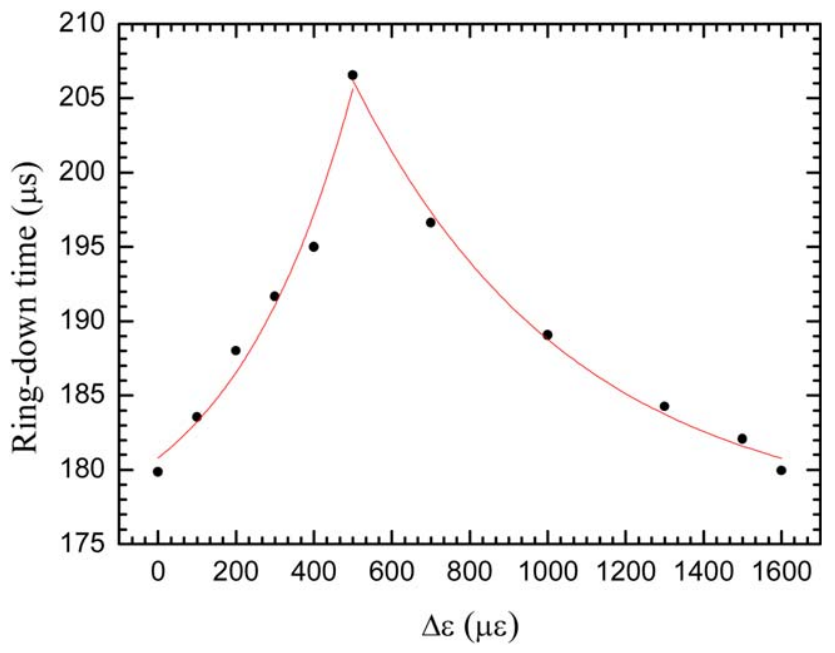

Figure 5 Cavity ring-down time versus applied strain to the chirpedFBG when interrogated by the OTDR. [Color figure can be viewed in the online issue, which is available at wileyonlinelibrary.com]

cavity and acted as a strain sensing device. Strain results could be attained with both conventional CRD-based configuration and the OTDR. Although measurements acquired with the conventional CRD configuration provided a better a ring-down time response $(22.8 \mu \mathrm{s})$, it was possible to prove the viability of using the OTDR as an interrogation method combined with the CRD configuration. Using the CRD configuration it was possible to obtain a linear response to strain applied to the chirpedFBG in the range $0-2000 \mu \varepsilon$, at the wavelength of $1570 \mathrm{~nm}$, and a sensitivity of $1.34 \mathrm{~ns} / \mu \varepsilon$ was obtained. In overall, the proposed configuration is simpler than the conventional CRD, that uses the laser and modulator setup, provides dual interrogation method and the OTDR also enables remote sensing.

\section{ACKNOWLEDGMENTS}

This work was financed by FCT-Fundação para a Ciência e Tecnologia (Portuguese Foundation for Science and Technology) and by ERDF (European Regional Development Fund) through: COMPETE Programme (Operational Programme for Competitiveness) within project FCOMP-01-0124-FEDER-037281; ON.2 - O Novo Norte (Northern Portugal Regional Operational Programme).

\section{REFERENCES}

1. G. Berden, R. Peeters, and G. Meijer, Cavity ring-down spectroscopy: Experimental schemes and applications, Int Rev Phys Chem 19 (2000), 565-607.

2. G. Stewart, K. Atherton, H. Yu, and B. Culshaw, An investigation of an optical fibre amplifier loop for intra-cavity and ring-down cavity loss measurements, Meas Sci Technol 12 (2001), 843-849.

3. R.S. Brown, I. Kozin, Z. Tong, R.D. Oleschuk, and H.-P. Loock, Fiber-loop ring-down spectroscopy, J Chem Phys 117 (2002), 10444-10447.

4. W.P. Tarsa, D.M. Brzozowski, P. Rabinowitz, and K.K. Lehmann, Cavity ring-down strain gauge, Opt Lett 29 (2004), 1339-1341.

5. N. Ni, C.C. Chan, X.Y. Dong, J. Sun, and P. Shum, Cavity ringdown long period fiber grating strain sensor, Meas Sci Technol 18 (2007), 3135-3138.

6. C. Wang and S.T. Scherrer, Fiber ring-down pressure sensors, Opt Lett 29 (2004), 352-354.

7. C. Wang, Fiber ringdown temperature sensor, Opt Eng Lett 44 (2005), 030503.
8. N. Ni, C. Chan, L. Xia, and P. Shum, Fiber cavity ring-down refractive index sensor, IEEE Photon Technol Lett 20 (2008), 1351-1353.

9. K.M. Zhou, D.J. Webb, C.B. Mou, M. Farries, N. Hayes, and I. Bennion, Optical fiber cavity ring down measurement of refractive index with a microchannel drilled by femtosecond laser, IEEE Photon Technol Lett 21 (2009), 1653-1655.

10. W.C. Wong, W. Zhou, C.C. Chan, X. Dong, and K.C. Leong, Cavity ring-down refractive index sensor using photonic crystal fiber interferometer, Sens Actuat B 161 (2012), 108-113.

11. P.B. Tarsa, A.D. Wist, P. Rabinowitz, and K.K. Lehmanna, Single cell detection by cavity ring-down spectroscopy, Appl Phys Lett 85 (2004), 4523-4525.

12. J.A. Barnes, M. Dreher, K. Plett, R. Brown, C.M. Crudden, H.-P. Loock, Chemical sensor based on a long period fibre grating modified by a functionalized polymethylsiloxane coating, Analyst 133 (2008), 1541-1549.

13. K.M. Zhou, D. Webb, M. Farries, N. Hayes, L. Zhang, and I. Bennion, Biochemical sensor based on a novel all-fibre cavity ring down spectroscopy technique incorporating a tilted fibre Bragg grating, Opt Lasers Eng 47 (2009), 1023-1027.

14. H. Waechter, J. Litman, A.H. Cheung, J.A. Barnes, and H.P. Loock, Chemical sensing using fiber cavity ring-down spectroscopy, Sensors 10 (2010), 1716-1742.

15. C.M. Rushworth, D. James, J.W.L. Lee, and C. Vallance, Top notch design for fiber-loop cavity ring-down spectroscopy, Anal Chem 83 (2011), 8492-8500.

16. G. Stewart, K. Atherton, and B. Culshaw, Cavity-enhanced spectroscopy in fiber cavities, Opt Lett 29 (2004), 442-444.

17. M. Fabian, E. Lewis, T. Newe, and S. Lochmann, Optical fibre cavity for ring-down experiments with low coupling losses, Meas Sci Technol 21 (2010), 094034

(C) 2015 Wiley Periodicals, Inc.

\section{DUAL-BAND METAMATERIAL-INSPIRED ANTENNA FOR MOBILE APPLICATIONS}

Sameer Kumar Sharma and Raghvendra Kumar Chaudhary

Department of Electronics Engineering, Indian School of

Mines-Dhanbad, Dhanbad 826004, Jharkhand, India; Corresponding author: raghvendra.chaudhary@gmail.com

Received 31 October 2014

ABSTRACT: In this letter, a metamaterial (MTM) inspired antenna is designed to operate at DCS, GSM1800, WCDMA, and Bluetooth and hence shows good potential to be used in mobile applications. The proposed antenna shows $n$ characteristics with first band $(1.61-1.84 \mathrm{GHz})$ resonant at $1.72 \mathrm{GHz}$ and second $(2.08-2.5 \mathrm{GHz})$ at $2.17 \mathrm{GHz}$ with substrate dimensions of $0.29 \lambda_{o} \times 0.29 \lambda_{o} \times 0.01 \lambda_{o}$. Percentage input impedance bandwidth $\left(S_{11}<-6 \mathrm{~dB}\right)$ is $13.4 \%$ for first and $19.4 \%$ for second band, respectively. The proposed antenna shows monopole-like radiation pattern in xz-plane while dipolar-type in yz-plane at both frequencies of operation, respectively. The measured gain of proposed antenna is 1.8 and $1.6 \mathrm{~dB}$ at 1.72 and $2.17 \mathrm{GHz}$ with radiation efficiency of 97.2 and $99.1 \%$, respectively. (C) 2015 Wiley Periodicals, Inc. Microwave Opt Technol Lett 57:1444-1447, 2015; View this article online at wileyonlinelibrary.com. DOI 10.1002/mop.29113

Key words: complimentary split ring resonator; dual-arm antenna; mobile antenna; metamaterials; partial ground

\section{INTRODUCTION}

Recent trend shows a lot of research is going on in the development of miniaturized mobile antennas. Planar microstrip patch antennas have been utilized in mobiles from a long time. With the introduction of slots, shortening pins, and different resonating 